\title{
Brucellosis: Review on the Recent Trends in Pathogenicity and Laboratory Diagnosis
}

\author{
Supriya Christopher, Umapathy B L, Ravikumar K L
}

Department of Microbiology, Kempegowda Institute of Medical Sciences, BSK II Stage, Bangalore, India

Address for correspondence: Mrs. Supriya C, E-mail: supriyachristopher@hotmail.com

\begin{abstract}
Brucellosis is a zoonotic infection transmitted from animals to humans by the ingestion of infected food products, direct contact with an infected animal or inhalation of aerosols. The last method is remarkably efficient given the relatively low concentration of organisms (10 - 100 bacteria) needed to establish infection in humans, and has brought renewed attention to this old disease. Brucella is a facultative intracellular pathogen that has the ability to survive and multiply in the phagocytes and cause abortion in cattle and undulant fever in humans. Brucella spp particularly $B$. melitensis, $B$. abortus, and $B$. suis represent a significant public health concern. At present, $B$. melitensis is the principle cause of human brucellosis in India. Molecular studies have demonstrated the phylogenetic affiliation of Brucella to Agrobacterium, Ochrobactrum, and Rhizobium. Human brucellosis still presents scientists and clinicians with several challenges, with regard to the understanding of its pathogenic mechanism, severity, progression, and development of improved treatment regimens. Molecular studies have now highlighted the pathogenesis of Brucella, for the development of newer diagnostic tools that will be useful in developing countries where brucellosis is a common, but often a neglected disease. This review compiles all these issues in general and the pathogenicity and newer diagnostic tools in particular.
\end{abstract}

Keywords: Brucellosis, lipopolysaccharide, virulence, zoonotic disease, serodiagnosis

DOI: $10.4103 / 0974-2727.72149$

www.jlponline.org

\section{INTRODUCTION}

Op rom the time of the Roman era, organisms resembling Brucellae have been detected in carbonized cheese. Brucellosis was predominant in the Mediterranean region and its history is associated with military campaigns. This disease was fully elucidated by Sir David Bruce, Hughes, and Zammit working in Malta. ${ }^{[1]}$ Bang discovered B. abortus, the causative agent of abortion in cattle and of brucellosis (undulant fever) in human beings. ${ }^{[2]}$ The disease remains the world's most common bacterial zoonosis, with over half a million new cases annually and the prevalence rate in some countries exceeds ten cases per 100,000 population, ${ }^{[3]}$ being higher in people working in organized farms. ${ }^{[4]}$ Despite being endemic in many developing countries, brucellosis is under-diagnosed and under-reported. ${ }^{[5]}$

Brucellosis in human beings is rarely fatal, but can lead to severe debilitation and disability. Nevertheless, it is reported that approximately $2 \%$ of the untreated patients die of brucellosis. ${ }^{[6]}$ The disease has the tendency toward chronicity and persistence, becoming a granulomatous disease capable of affecting any organ system. ${ }^{[7]}$ The timely and accurate diagnosis of human brucellosis continues to challenge clinicians because of its non-specific clinical features, slow growth rate in the blood culture, and the complexity of its serodiagnosis. ${ }^{[8,9]}$

Phylogenetically, Brucella is classified within the $\alpha 2$ subdivisions of the Proteobacterium, which includes Agrobacterium, Rickettsia, Rhodobacterium, and Rhizobium. ${ }^{[10]}$ Establishing a relationship within the genus has been challenging because of the relatively few genetic polymorphisms that distinguish each species. ${ }^{[11]}$ Six species are recognized within the genus Brucella: B. abortus, B. melitensis, B. suis, B. ovis, B. canis, and B. neotomae. This classification is based on the differences in pathogenicity and host preference. ${ }^{[12]}$

In recent times, two new species have been added to this genus, $B$. cetaceae and B. pinnipediae, isolated from marine mammals, cetaceans, and pinnipeds. ${ }^{[13]}$ The Brucella genome consists of two circular chromosomes, without plasmids, suggesting a remarkable difference compared to the single chromosome of many bacteria. 
Successful infection by pathogenic bacteria often depends on their ability to survive and multiply within the host cells. To do so, they alter or adapt to the host cell environment. To these ends, pathogenic bacteria contain a variety of secretion systems, including type I, II, III \& IV systems which can export virulence factors to the environment or into the infected host cell. ${ }^{[14]}$ However some of the Brucella spp lack these secretion system, except for some like B. melitensis contains genes for flagellum- specific type III and IV secretion systems. ${ }^{[15]}$ These secretion systems are involved in variety of process ranging from the delivery of virulence factors into the eukaryotic cell to conjugation, transfer of genetic material, uptake or release of DNA. ${ }^{[1]]}$ The recent completion of B. melitensis (Gene Bank NC003317) and (NC003318), ${ }^{[17]}$ B. suis (Gene Bank NC002969), and the B. abortus ${ }^{[18]}$ genome sequence projects have provided tremendous information for understanding the mechanisms of Brucella pathogenicity. The availability of the complete genome sequences and advancement of genomics and proteomics has enabled scientists to understand the disease and its pathogenic mechanisms. The development in culture and serological methods are routinely used for the diagnosis of the disease, however, advanced molecular detection and typing methods have contributed to improving the laboratory diagnosis. This article reviews and summarizes the current knowledge of the pathogenic mechanisms and the newer diagnostic advances made in human brucellosis.

\section{PATHOGENICITY}

Brucella spp are facultative intracellular bacteria that have the ability to avoid the killing mechanism and proliferate within the macrophages, similar to other intracellular pathogens.

To be a successful infectious agent, Brucella requires four steps: adherence, invasion, establishment, and dissemination within the host

Opsonised and non opsonised Brucella can infect macrophages. Thereby indicating direct host cell contact which allows adherence and invasion as well as antibody or complement mediated phagocytises. In the macrophages. Brucella cells survive and multiply, inhibiting phagosome -lysososme fusion. Finally, the accumulated bacteria are disseminated to other host cells. ${ }^{[15]}$

After infecting the host, the pathogen becomes sequestered within the cells of the reticuloendothelial system. The mechanism by which Brucella enters the cells and evades intracellular killing and the host immune system is a subject of much research and debate.
Several studies on the virulence factors are directed at the main components of the outer membrane. The outer membrane contains Lipopolysaccharide (LPS), which is the major virulence factor of Brucella. It possesses a peculiar non-classical LPS as compared to the classical LPS from Enterobacteria, such as Escherichia. coli ${ }^{[19]}$ [Table 1].

Smooth LPS has a role in cell entry and immune evasion of the infected cell. It also alters the capacity of the infected cell to present foreign antigens to the MHC class II antigen presentation system, hence, preventing the attack and killing the infected cell with the help of the immune system. ${ }^{[20]}$ LPS has three domains:

Lipid A, the core oligosaccharide, and the O-antigen.

The O-polysaccharide of the smooth type Brucella LPS is an unbranched homopolymer of 1-2 linked 4, 6 dideoxy4 -formamido and $\alpha$-D mannopyranosyl, usually with an average chain length of 96 to100 glycosyl subunits. ${ }^{[21]}$

The O-polysaccharide is linked to the core polysaccharide composed of mannose, glucose, 2-amino-2, 6-dideoxyD-glucose, 2-amino-2-deoxy-D-glucose, 3 deoxy-Dmanno-2-octulosonic acid (KDO), and unidentified sugars. (The lipid A linked to the core polysaccharide contains 2, 3-diamino-2,3 dideoxy-D-glucose as the backbone and amide- and ester-linked long chain saturated $\left(\mathrm{C}_{16: 0}\right.$ to $\left.\mathrm{C}_{18: 0}\right)$ and hydroxylated fatty acids. ${ }^{[22]}$

The heterogeneity of the enterobacteria is known to be related to the length of its O-polysaccharide and different chemical substitutions in the core oligosaccharide and lipid-A. ${ }^{[23]}$ In the enterobacterial lipid A, the degree of heterogeneity depends on the different combinations in which the amide- and ester-linked fatty acid, phosphates, neutral sugars, ethanolamine, and different types of backbone amino sugars occur in the molecule, ${ }^{[24]}$ whereas, in Brucella lipid A, the degree of heterogeneity depends mainly on various fatty acid substitutions. There is an absence of backbone constituents and ester-linked acyl-oxyacyl residues in Brucella lipid A, as compared to enterobacterial lipid A. ${ }^{[25]}$ Determination of heterogeneity

\begin{tabular}{ll}
\hline $\begin{array}{l}\text { Table 1: Difference between Classical \& Non- } \\
\text { classical LPS }\end{array}$ \\
\hline Classical LPS & Non classical LPS \\
Exhibit high toxicity & Exhibit low toxicity for endotoxin \\
& sensitive mice and rabbit \\
High pyrogenicity & Low pyrogenicity \\
Inducers of interferons and tumor & Weak inducers of interferons and \\
necrosis factor & tumour necrosis factor \\
Examples: E. coli & Example: Brucella. abortus \\
\hline
\end{tabular}

Journal of Laboratory Physicians / Jul-Dec 2010 / Vol-2 / Issue-2 
in Brucella LPS is important for practical purposes, as it is the most relevant antigen during infection and vaccination. The genome sequences of $B$. melitensis, B. suis, and $B$. abortus have become available recently. ${ }^{[26]}$ They are similar in sequence, organization, and structure. Comparative genomics provides an insight into the aspects of Brucella virulence that has only been suspected earlier. Using the complete genome sequence of $B$. melitensis, Dricot et al. ${ }^{[27]}$ has generated a database of protein coding ORFs and constructed an ORFeome library of 3091 gateway entry clones, each containing a defined ORF. The genome sequence of the B. melitensis strain 16M contains 3,294,935 $\mathrm{bp}$, which is distributed over two circular chromosomes of 2,227,144 bp and 77,787 bp, encoding 3,197 ORFs. The genome of B. abortus biovar (strain 9-941) has $3.3 \mathrm{Mb}$ composed of two circular chromosomes of 2,124,242 (Chr I) and 1,162,780 bp (Chr II). ${ }^{[28]}$ The genome of B. suis is a 1330 genome consisting of two circular chromosomes of $2,107,7892$ bp and 1,207,381 bp. ${ }^{[29]}$

The genome of $B$. abortus shares more fragments with B. suis and B. melitensis, than B. suis and B. melitensis do with each other. A majority of the genes studied are involved in Brucella biosynthesis and O-chain synthesislike persominesynthetase (per), mannosyl tranferase (wbkA,WbdA,B,C), phosphoglucomutase (pgm), ABC type transporters $\left(\mathrm{W}_{2 \mathrm{~m}}, \mathrm{~W}_{2 \mathrm{f}}\right)$, and mannose (man $\left.\mathrm{A}, \mathrm{B}, \mathrm{C}\right)$. The BvrR / BvrS gene sensing system acts as a cascade of protein phoshorylation to modulate the key expression, and these key factors are involved in cell binding and penetration. This system has an effect on the expression of cell surface proteins Omp 25 (Omp3a) and Omp 229 (Omp3b). ${ }^{[30]}$

This altered cell expression of the surface proteins allows Brucella to bind to and penetrate the lysosomal pathway. A type IV secretion system (Vir B) selectively transports proteins and macromolecules through the membranes and is essential for intracellular survival, in case of Brucella. It also helps in adherence of the bacterium to the host cell and cell entry. ${ }^{[31]}$ A large number of attenuated mutants, with structural defects in their Lipopolysaccharide, confirm the importance of this molecule in Brucella virulence. ${ }^{[32]}$ Heat shock protein 60 (Hsp60), a member of the GroEl family of chaperonins, is expressed on the cell surface of wildtype Brucella spp, but not on VirB mutants. Hsp60 seems to play a part in cell adherence by binding to a cellular prion molecule called PrPr. As the exportation of Hsp60 is VirBdependent, it has been postulated that Hsp60 may in fact be a virulence factor. ${ }^{[33]}$ Once the organism binds to the macrophages, the internalization vesicles that would fuse with the endosomes take it up. The endosomes are lysed by acidification. This acidification is thought to induce Vir B expression. ${ }^{[34]}$

\section{DIAGNOSIS}

Diagnostic methods for brucellosis are primarily based on serology, with the LPS smooth chains producing the greatest immunological responses in various hosts. The major diagnostic problem is due to the similarity of the O-antigenic side chain of LPS of Brucella and other organisms like Yersinia enterocolitica O : 9, Vibrio. cholerae, Esherichia. coli $0: 157$, and Francisella. tularensis. Alternative antigens have been evaluated for their diagnostic potential, for a possible improvement in its specificity, however, these have largely been unsuccessful. (Blood culture is the gold standard in the diagnosis of bacterial infections including brucellosis, but this method is successful in only $40-$ $70 \%$ of the cases. The Biphasic Ruiz-Castaneda system is the traditional method for the isolation of Brucella sps in clinical samples. ${ }^{[35]}$ It has been largely replaced by the lysis centrifugation technique, where a higher rate of positive blood culture has been reported. An automated culture system has also improved the speed of detection. ${ }^{[36]}$ Bone marrow cultures may provide higher sensitivity, yield faster culture times, and may also be superior to blood culture, when evaluating patients with previous antibiotic use. Brucella can also be cultured from pus, tissue, cerebrospinal fluid (CSF), and pleural / joint / ascitic fluid. ${ }^{[37]}$

However, the results have not yet been universally reproducible.

\section{SERODIAGNOSIS}

In the absence of culture facilitates the diagnosis of brucellosis relies on agglutination tests, such as, the Rose Bengal test, serum agglutination test, the antiglobulin or Coombs test, complement fixation test, and the recently introduced immunocapture test.

The Rose Bengal test is used as a screening test and positive results are confirmed by the serum agglutination tests. ${ }^{[38]}$ This agglutination test is based on the reactivity of antibodies against the smooth lipopolysaccharide. In the Rose Bengal Plate (RBPT) agglutination test the sensitivity is high $(>99 \%)$ and false negative results are rarely observed. To increase the specificity the test may be applied to a serial dilution (1:2 through 1:64) of the serum samples. ${ }^{[39]}$ The Standard Tube Agglutination Test (SAT) developed by Wright and colleagues remains the most popular and easy test to perform. SAT can measure the total 
quantity of the agglutinating antibodies (IgG and $\operatorname{IgM}$ ). The quantity of specific $\operatorname{IgG}$ is determined by treatment of the serum with $0.005 \mathrm{M} 2$ mercaptoethanol (2ME), which inactivates the agglutinability of the IgM. However, many patients have low levels of agglutinating IgG antibodies and the results can easily be misinterpreted. SAT titers above $1: 160$ are considered diagnostic in conjunction with a compatible clinical presentation, however, in endemic areas the titer of $1: 320$ is taken as the cut off. Coomb's test is the most suitable and sensitive test for confirmation in relapsing patients with persisting disease, but it is complex and demands technique. Enzyme linked immunosorbant assay (ELISA) has become increasingly popular, as well as a standardized assay for brucellosis. It measures $\operatorname{IgG}$, $\operatorname{IgM}$, and $\operatorname{IgA}$, which allows a better interpretation of the clinical situation. The specificity of ELISA, however, seems to be less than the agglutination tests. As the diagnosis of Brucella is based on the detection of antibodies against smooth LPS, the cut-off value needs to be adjusted, to optimize the specificity when used in endemic areas. ${ }^{[40]}$ ELISA can also be applied in the diagnosis of CNS brucellosis with varying success and further research must be aimed at improving the diagnosis of this condition. The Fluorescence polarization assay (FPA) offers a valuable alternative to conventional serological tests. This assay measures the size of a florescent tagged molecule such as an antigen - ideally antigens selected for this technique should be small $(20 \mathrm{Kda})$. The utilization of the O-side chain of LPS from Brucella spp has shown encouraging results. ${ }^{[41]}$ The sensitivity of this test at the selected cut-off value is $96 \%$ for culture-confirmed brucellosis and the specificity is $98 \%{ }^{[42]}$

Immunochromatographic Brucella IgM / IgG lateral flow assay (LFA), a simplified version of ELISA has a great potential as a rapid point-of-care assay. Studies have shown that this test has high sensitivity and specificity for Brucella IgM and IgG. This system uses a drop of blood obtained by a finger prick, which is used by the bedside and easy to interpret. It is a rapid and simple diagnostic test for confirmation of brucellosis in an endemic area. ${ }^{[43,44]}$ In recent years new immunocapture agglutination for antiBrucella (Brucella Capt BCAP) has been developed, to detect agglutinating and non-agglutinating antibodies with high sensitivity. It has been suggested as a possible substitute for Coombs test and a better marker for disease activity. ${ }^{[45]}$

\section{MOLECULAR DETECTION}

Over the past decade there has been a major advancement in all aspects of molecular diagnostics with regard to human brucellosis. Polymerase chain reaction (PCR)-based tests are proving to be faster and more sensitive than the traditional methods.

Several genus-specific PCR systems using primer pairs that target 16SRNA sequences and genes of different outer membrane proteins have been developed (QueipoOrtuno and co-workers found 100\% sensitivity and 98.3\% specificity by using a B4 / B5 primer and amplifying a 223-bp fragment of the bcsp31 gene compared with 70\% constituents of blood culture. ${ }^{[46]}$

Incorporation of a robust DNA extraction method, such as the diatom-guanidinium isothiocyanate method, effectively removes the inhibitors commonly present in a variety of clinical specimens and may improve the sensitivity and reproducibility. ${ }^{[47]}$ However, as these PCR systems carry a high risk of contamination and require equipments for visualization, they are less suitable for routine diagnosis purposes. Hence, real time PCR systems have been developed that are faster and less prone to contamination and are thus more clinically useful. ${ }^{[48]}$

Relapsing brucellosis is another diagnosis challenge where PCR may prove to be useful. Nowadays, this is also used to assess treatment efficacy. ${ }^{[49]} \mathrm{PCR}$ is also useful in species differentiation and biotyping of isolates. There are some short nucleotide repeat sequences that are present in the Brucella genome showing a wide variation in the number of repeats between species and isolates. PCR amplification of these variable repeats is more robust than the classic typing methods for species and biovar identification.

This application could be applied epidemiologically to trace infections to specific flocks or dairy producers. One of the main characteristics of brucellosis is its marker tendency to relapse after completion of the treatment. This problem results from the intracellular location of the Brucella spp, which protects the bacteria from some of the basic mechanisms of the immune system, as well as from therapy. Relapses most frequently occur within six months to as long as two years of the initial treatment. Hence, it is necessary to monitor the patients during 12 months of the treatment. ${ }^{[0]}$

Morta and coworkers recently evaluated the usefulness of a PCR-based assay in a post-treatment follow up and relapse of patients with brucellosis. ${ }^{[51]}$ There are several PCR assays for the detection of Brucella DNA using pure culture, animal, and human clinical samples. However, the sensitivity and specificity of PCR for Brucella varies between laboratories, and hence standardization is required. ${ }^{[52]}$ 


\section{CONCLUSION}

Interest shown by the scientific community toward brucellosis has benefited developing countries like India. The DNA-DNA hybridization studies revealed a high degree of homology shared by six recognized species of Brucella. The genomic rearrangements, species-specific DNA sequence, and distinct patterns of gene inactivation suggest that $B$. abortus and B. melitensis share the same lineage, which differs from the B. suis lineage, which has undergone fewer genetic mutations, as it diverged from the most recent common ancestor of all Brucella. Although there are newer insights to the pathogenesis of Brucella spp, a lot of development is needed in the aspect of treatment, as Brucella spp do not follow the classical method of virulence, which has made investigation in this area slower. As brucellosis poses health threats to humans, and morbidity in untreated diseases is substantial, thus early consideration and diagnosis of brucellosis is important. As brucellosis is often misdiagnosed or overlooked, physicians in both endemic and nonendemic areas must be aware in their diagnosis of febrile diseases.

\section{REFERENCES}

1. Cultuer SJ, Whatmore AM, Commander NJ. Brucellosis- new aspects of an old disease. J Appl Microbiol 2005;98:1270-81.

2. Bang B. The etiology of epizootic abortion. J Comp Pathol Therap 1897;10:125.

3. Mantur BG, Amarnath S. Brucellosis in India. J Biosci 2008;33:539-47.

4. Umapathy BL, Nagamani M, Bhat P, Keshavamurthy BS. Seroepidemiological studies on human brucellosis in and around Bangalore. Indian J Comp Microbiol Immunol Infect Dis 1984;5:83-7

5. Godfroid J, Cloekaert A, Liautard JP. From the discovery of the Malta fever's agent to the discovery ofa marine mammal reservoir, brucellosis has continuously been re-emerging zoonosis. Vet Res 2005;36:313-26.

6. Madkour MM, Madkoure. Brucellosis. New York: Springer Verlag; 2001. p. 11.

7. Pappas G, Papadimitriou P, Akritidis N, Christou L, Tsianos EV. The new global map of human brucellosis. Lancet Infect Dis 2006;6:91-9.

8. Colmenero JD, Reguera JM, Cabrera FP. Serology, clinical manifestations and treatment of brucellosis. Infection 1990;18:152-56.

9. Memish Z, Mah MW, Al Mahmoud S, Al Shaalan M, Khan MY. Brucella bacteremia: clinical and laboratory observations in 160 patients. J Infect Dis 2000;40:59-63.

10. Morenoe EE, Stackebrandt E, Dorsch M, Wolters J, Busch M, Mayer II. Brucella. abortus 16Sr RNA and lipid A reveal a phylogenetic relationship with members of the $\alpha 2$ subdivision of the class proteobacteria. J Bacteriol 1990;172:3569-76.

11. Corbel MJ, Brinley Morgan WJ. Genus Brucella. In: Bergey's Manual of systematic Bacteriology. Kreig Nr, Holt JG, editors. Baltimore: Williams \& Wilkins; 1984. p. 377-88.

12. Cloeckaert A, Grayon M, Grepinet O, Boumedine KS. Classification of Brucella stains isolated from marine mammals by infrequent restriction site -PCR and development of specific PCR identification tests. Microbes Infect 2003;5:593-602.

13. Michaux C, Bang SG, Jumas BE, Guigue T, Allardet A, O' Callaghan D, et al. Genome structure \& phylogeny in the genes Brucella. J Bacteriol 1997;179:3244-49.

14. Harze WD. Brucella virulence. Genome biology; 2002. p. 3.
15. Ko J, Splitter GA. Molecular host interaction in brucellosis: Current understanding and futureapproaches to vaccine development for mice and humans. Clin Microbial Rev 2003;16:65-78.

16. Vidya C, Remi F, Stephane D, Nora C, Jorge N, Gabriel W. Structure of the outer membrane complex of a type IV secretion system. Nature 2009;462:1011-6.

17. DelVecchio VG, Kapatral V, Redkar RJ, Patra G, Mujer C, Los T, et al. The genome sequence of the facultative intracellular pathogen Brucella. melitenisis. Proc Natt Acad Sci USA 2002;99:443-8.

18. Sanchez D, Zandomeni RO, Cravero S, Verdun RE, Pierrou E, Faccio P, et al. Gene discovery through genomic sequencing of Brucella. abortus. Infect Immun 2001;69:865-8.

19. Lapaque N, Moriyon I, Moreno E, Grovel JP. Brucella lipopolysaccharide acts as a virulence factor. Curr Opin Microbiol 2005;8:60-6.

20. Araya IN, Elzer P, Rowge, Enright FM, Winter J. Temporal development of protective cell mediated immunity \& humoral immunity in BALB / CMICE infected with Brucella abortus in $\mathrm{J} 74$ Macrophages. Infect Immun 1989;143:3330-7.

21. Bundle DR, Cherwonogrodzky JW, Gidney MAJ, Meckll RJ, Perry MB, Peters T. Definition of Brucella A \& M epitopes by monoclonal typing reagents and synthetic oligosaccharides. Infect Immun 1989;57:2829-39.

22. Monero E, Borowiak D, Mayer H. Brucella Lipopolysaccharides and polysaccharides. Ann Inst Pasteur Microbiol 1987;138:102-5.

23. Freer E, Roja SN, Weintraub A, Lindberg AA, Moreno E. Heterogeneity of Brucella. abortus lipopolysaccharides. Res Micrbiol 1995;146:569-78.

24. Raetz CR. Biochemistry of endotoxins. Ann Rev Biochem 1990;59: $129-70$.

25. Diaz AE, Aragon V, Marin C, Alonso B, Tont M, Moreno E. Comparative analysis of Brucella Serotypes A \& M And Yersinia enterocolitica 0:9 polysaccharides for serological diagnosis of brucellosis in cattle, sheep and goats. J Clin Microbiol 1993;31:3136-41.

26. Halling SM, Peterson BD, Bricker BJ, Zuernei RL, Quing Z, et al. Completion of the genome sequence of Brucella abortus and comparision to the similar genomes of Brucella. melitensis and Brucella. suis. J Bacteriol 2005;8: 2715-26.

27. Dricot A, Rual JF, Lamesch P, Beetm N. Generation of the Brucella melitensis ORFeome version 1. Genome Research 2004;4:2201-06.

28. Patricia G, Cardosa, Gilson Costa CM, Vasco A, Sergi CO. Brucella sps non conocial LPS: Structure, interaction with host immune system. Microbial cell factories 2006;5:131-11.

29. Vizcaino N, Cloeckaert A, Zygmunt MS, Fernandez-Lago L. Characterization of a Brucella species 25-kilobase DNA fragment detected from Brucella abortus reveals a large gene cluster related to the synthesis of a polysaccharide. Infect Immune 2001;69:6738-48.

30. Lopez G, Guzman V, Manterola L. Regulation of Brucella virulence by two component system BvrR / BvrS. Vet Microbiol 2002;90:329-39.

31. Maria PF, Maximillan M, Robert HG, Henk LS. Human brucellosis. Lancet Infect Dis 2007;7:775-86.

32. Arenas GN, Staskevish AS, Aballay A. Intracellular tracffiking of B abortus in J774 macrophages. Infect Immun 2000;68:4255-63.

33. Wataria M. Interaction between Brucella abortus and cellular prion protein in lipid raft microdomains. Microbes Infect 2004;6:93-100.

34. Kittelberger R, Hilbink F, Hansen MF, Ross GP, Joyce MA. Serological cross reactivity between B. abortus and Yersinia enterocolitica 0:9 II the use of Yersinia outer proteins for specific detection of Yersinia enterocolitica infections in ruminants. Vet Microbiol 1997;57:271-80.

35. Ruiz CM. A practical method for routine blood culture in brucellosis. Proc Soc Exp Biol Med 1954;86:154-5.

36. Gotuzz E, Carrillo C, Guersa J, Llosa L. An evaluation of diagnostic methods for brucellosis - the value of bone marrow culture. J Infect Dis 1986;153:122-5.

37. Mantur BG, Biradar MS, Bidri C, Mallana S, Verrappa K, et al. Protean clinical manifestation \& diagnostic challenges of human brucellosis in adults: 16 yrs experiancein an endemic area. J Med Microbiol 2006;55:897-903.

38. Ruiz JD, Sanchez G, Rehuera JM, Martin L, Lopez P, Colmenew JD. Rose Bengal test diagnosis of human bucellosis in emergency department in endemic areas. Clin Microbial Infect 2005;11:221-5. 


\section{Christopher, et al.: Pathogenicity and laboratory dignosis of brucellosis}

39. Ruiz-Mesa JD, Sanchez-Gonzalez J, Reguera JM, Martin L, Lopez-Palmero S. Diagnostic yield and use for the rapid diagnosis of human brucellosis in emergency departments in endemic areas. Clin Microbiol Infect 2005;11:221-5.

40. Smith HL, Kadri SM. Brucellosis in India: A deceptive infectious disease. Indian J Med Res 2005;122:375-84.

41. Nielsen K, Lin M, Gall D, Jolley M. Flourescence polarization immunoassay: detection of antibody of Brucella abortus. Methods 2000;22:71-6.

42. Lucero NE, Escobar GI, Ayala SM, Silva PN. Fluorescence polarization assay for diagnosis of human brucellosis. J Med Microbial 2003;52:883-7.

43. Hasanjani RMR, Soleimani Amin MJ, Abdoel TH, Sits HL. Application of a rapid and simple Brucella-specific IgM and IgG antibody test for the serodiagnosis of brucellosis in a hospital in Iran. Trans R Soc Trop Med Hyg 2005;9:744-50.

44. Hasan I, Turan B, Omer E, Hayrettin A, Pekcan D, Theresia H, et al. Use of the Brucella $\operatorname{IgM}$ and $\operatorname{IgG}$ flow assays in the serodiagnosis of human brucellosis in an area endemic for brucellosis. Am J Trop Med Hyg 2004;70:688-94.

45. Casanova A, Ariza J, Rubio M, Masuet C, Díaz R. Brucella capt versus classical tests in serological diagnosis and management of human brucellosis. Clin Vaccine Immunol 2009;16:844-51.

46. Queipo-Ortuño MI, Colmenero JD, Baeza G, Morata P. Comparision between light cycler real time polymerase chain reaction (PCR) assay with serum and PCR, enzyme linked immunosorbant assay with whole blood samples for the diagnosis of human brucellosis. Clin Infect Dis 2005;40:260-4.

47. Boom R, Sol CJ, Salimans MM. Rapid and simple method for purification of nucleic acids. J Clin Microbiol 1990;28:495-503.

48. Debaeumont C, Falconnet PA, Maurin M. Real time PCR for detection of Brucella Spp DNA in human serum samples. Eur J Clin Microbiol Infect Dis 2005;24:842-5.

49. Nimri LF. Diagnosis of recent and relapsed cases of human brucellosis by PCR assay. BMC Infect Dis 2003;3:5.

50. Keil FW, Khan NJ. Analysis of 506 consecutive positive serologic tests for brucellosis in Saudi Arabia. J Clin Microbiol 1987;25:1384-7.

51. Morta P, Queiyo -ortuno ML, Rodrignej TA, Ordina A. Specificity of a polymerase chain reaction assay of a target sequence on the 31 Kilodalton Brucella antigen DNA used to diagnose human Brucellosis. Eur J Clin Microbiol Infect Dis 2001;20:27-31.

52. Elena N, Maria C, Javier S. Diagnosis of human brucellosis using PCR. Expert Rev Mol Diag 2004;4:115-23.

Source of Support: Nil, Conflict of Interest: None declared. 